\title{
A family of graphs that are determined by their normalized Laplacian spectra *
}

\author{
Abraham Berman, \\ Department of Mathematics, \\ Technion-Israel Institute of Technology, Haifa 32000, Israel \\ Dong-Mei Chen, Zhi-Bing Chen \\ College of Mathematics and Statistics, \\ Shenzhen University, Shenzhen 518060, P.R. China \\ Wen-Zhe Liang, Xiao-Dong Zhang ${ }^{\dagger}$ \\ School of Mathematical Sciences, MOE-LSC, SHL-MAC \\ Shanghai Jiao Tong University, Shanghai 200240, P. R. China
}

\begin{abstract}
Let $F_{p, q}$ be the generalized friendship graph $K_{1} \bigvee\left(p K_{q}\right)$ on $p q+1$ vertices obtained by joining a vertex to all vertices of $p$ disjoint copies of the complete graph $K_{q}$ on $q$ vertices. In this paper, we prove that $F_{p, q}$ is determined by its normalized Laplacian spectrum if and only if $q \geq 2$, or $q=1$ and $p \leq 2$.

AMS Classification: 05C50, 05C35

Key words: Normalized Laplacian spectrum; generalized friendship graph; spectral characterization; cospectral graph.
\end{abstract}

\section{Introduction}

Spectral graph theory studies the relations between the structure of a graph and eigenvalues of matrices associated with it. One of the main problems in spectral graph theory is which graphs are determined by their spectrum or equivalently, finding nonisomorphic graphs $G$ and $H$ that have the same spectrum. Many results on these questions can be found in two excellent surveys (see [9, 10]) by Van Dam and Haemers.

*This work is supported by the Joint NSFC-ISF Research Program (jointly funded by the National Natural Science Foundation of China and the Israel Science Foundation (Nos. 11561141001, 2219/15), the National Natural Science Foundation of China (No.11531001).

$\dagger$ Corresponding author. E-mail: xiaodong@sjtu.edu.cn 
Let $G$ be an undirected simple graph with vertex set $V(G)$ and edge set $E(G)$. Let $d_{v}$ be the degree of a vertex $v \in V(G)$. The normalized Laplacian matrix of a graph $G$ is defined to be $\mathcal{L}(G)=\left(l_{u v}\right)$, where

$$
l_{u v}= \begin{cases}1, & \text { if } u=v \text { and } u \text { is not an isolated vertex; } \\ -\frac{1}{\sqrt{d_{u} d_{v}}}, & \text { if } u \text { is adjacent to } v \\ 0, & \text { otherwise. }\end{cases}
$$

The eigenvalues of $\mathcal{L}(\mathcal{G})$ are called $\mathcal{L}$-eigenvalues. This paper deals with the normalized Laplacian matrix of $G$, so we denote its spectrum of $G$ (the all eigenvalues $\mathcal{L}(G)$ of $G$, including multiplicities) by $S p(G)$. We say that $G$ and $H$ are cospectral if they are not isomorphic, but $S p(G)=S p(H)$, and that $G$ is determined by its normalized Laplacian spectrum if $S p(H)=S p(G)$ only when $H$ is isomorphic to $G$.

Chung in [4] showed how the normalized Laplacian spectrum reveals fundamental properties and structure of a graph. Butler [1] surveyed algebraic aspects of $\mathcal{L}(G)$ and provided (see [2] and [3]) several methods of constructing cospectral graphs. Almost all small graphs are determined by their normalized Laplacian spectrum (see [3]). The normalized Laplacian spectrum of a complete graph $K_{n}$ is $0, \frac{n}{n-1}$ with multiplicities 1 and $n-1$ respectively, and $K_{n}$ is determined by this spectrum [3].

Butler [1] conjectured that the only graphs cospectral with a cycle are $K_{1,3}$ and the graph $\gamma_{4 k}$ obtained by identifying the center vertex of a path on $2 k+1$ vertices and a vertex of a cycle on $2 k$ vertices; i.e., a cycle on $n$ vertices is determined by its normalized Laplacian spectrum if and only if $n>4$ and $4 \nmid n$. In general, up to now, there are very few graphs that are known to be determined by their normalized Laplacian spectrum. In this paper, we present a family of graphs that are determined by their spectrum.

Denote by $F_{p, q}$ the graph $K_{1} \bigvee\left(p K_{q}\right)$ on $p q+1$ vertices obtained by joining a vertex to all vertices of $p$ disjoint copies of the complete graph $K_{q}$ on $q$ vertices. The friendship graph $F_{k}$ consists of $k$ edge-disjoints triangles that meet in one vertex (for the famous friendship theorem, see [6]). Liu et. al. [8] proved the $F_{k}$ is determined by its Laplacian spectrum (see also [7]). Wang et. al. [12] proved that $F_{k}$ is determined by its signless Laplacian spectrum. Recently, Cioabă et. al. [5] proved that $F_{k}$ is determined by its adjacent spectrum if and only if $k \neq 16$, and that for $F_{16}$, there is only one graph nonisomorphic to $F_{16}$, but with the same adjacency spectrum. The friendship graphs are a subfamily of $F_{p, q}$, since $F_{k}=F_{k, 2}$.

In this paper we show that $F_{p, q}$ is determined by its normalized Laplacian spectrum if and only if $q \geq 2$, or $q=1$ and $p \leq 2$. This shows of course that the friendship graphs are determined by their normalized Laplacian spectrum.

Theorem 1.1. If $q \geq 2$, or $q=1$ and $p \leq 2$, then $F_{p, q}$ is determined by its normalized Laplacian spectrum. If $q=1$ and $p \geq 3$, then there is a graph $G$, not isomorphic to $F_{p, q}$ such that $S p(G)=S p\left(F_{p, q}\right)$.

Corollary 1.2. The friendship graph is determined by its normalized Laplacian spectrum 


\section{Proof of Theorem 1.1}

In order to prove the main results, we need the following lemmas.

Lemma 2.1. (i). If $p=1$, then the normalized Laplacian spectrum of $F_{1, q}$ is $0,1+\frac{1}{q}$ with multiplicities 1 and $q$ respectively.

(ii). If $p$ is a positive integer with $p \geq 2$, then the normalized Laplacian spectrum of $F_{p, q}$ is $0, \frac{1}{q}, 1+\frac{1}{q}$ with multiplicities $1, p-1$ and $p q-p+1$ respectively.

Proof. (i). Since $p=1, F_{1, q}=K_{q+1}$ and the assertion holds by [3].

(ii). If $q=1$, then $F_{p, q}$ is the star graph $K_{1, p}$ and its normalized Laplacian spectrum is $0,1,2$ with multiplicities $1, p-1$ and 1 respectively. If $q \geq 2$, then the normalized Laplacian spectrum of $K_{q}$ is $0, \frac{q}{q-1}$ with multiplicities 1 and $q-1$ respectively. By Proposition 3 in [3], the normalized Laplacian eigenvalues of $F_{p, q}$ is $0, \frac{1}{q}, 1+\frac{1}{q}$ with multiplicities $1, p-1$ and $p q-p+1$ respectively. Hence (ii) holds.

Lemma 2.2. If $q=1$ and $p \leq 2$, then $F_{p, q}$ is determined by its normalized Laplacian spectrum. If $q=1$ and $p \geq 3$, then there is a graph $G$, not isomorphic to $F_{p, q}$ such that $\operatorname{Sp}(G)=\operatorname{Sp}\left(F_{p, q}\right)$.

Proof. If $q=1$ and $p \leq 2$, clearly, $F_{p, q}$ is $K_{2}$ or $K_{1,2}$. So $F_{p, q}$ is determined by its normalized Laplacian spectrum. If $q=1$ and $p \geq 3$, then by [3], the normalized Laplacian spectrum of $F_{p, q}$ is the same as that of $K_{r, s}$, where $r+s=q+1$. So there is a graph $G$, not isomorphic to $F_{p, q}$ such that $\operatorname{Sp}(G)=\operatorname{Sp}\left(F_{p, q}\right)$.

From now, we assume that $p \geq 2$ and $q \geq 2$. In order to characterize graphs with three normalized Laplacian eigenvalues, for a simple connected graph $G$, denote by

$$
\begin{aligned}
\widehat{d}_{u} & :=\sum_{v \sim u} \frac{1}{d_{v}} \text { for } u \in V(G) ; \\
\widehat{\lambda}_{u v}: & =\sum_{w \sim u, w \sim v} \frac{1}{d_{w}} \text { for } u \sim v ;
\end{aligned}
$$

and

$$
\widehat{\mu}_{u v}:=\sum_{w \sim u, w \sim v} \frac{1}{d_{w}} \text { for } u \nsim v,
$$

where $u \sim v(u \nsim v)$ means that $u$ and $v$ are (not) adjacent in $G$. It follows from Theorem 1 in [11] that the following assertion holds.

Lemma 2.3. Let $G$ be a simple connected graph with $m$ edges and let $q \geq 2$ be a positive integer. Then $G$ has three distinct $\mathcal{L}$-eigenvlaues $0, \frac{1}{q}, 1+\frac{1}{q}$ if and only if the following three properties hold.

$$
\widehat{d}_{u}=\frac{(q+1) d_{u}^{2}}{2 m q^{2}}+\frac{(q-1) d_{u}}{q^{2}}, \quad u \in V(G) ;
$$




$$
\begin{gathered}
\widehat{\lambda}_{u v}=\frac{(q+1) d_{u} d_{v}}{2 m q^{2}}+\frac{q-2}{q}, \quad u \sim v ; \\
\widehat{\mu}_{u v}=\frac{(q+1) d_{u} d_{v}}{2 m q^{2}}, u \nsim v .
\end{gathered}
$$

Lemma 2.4. Let $G=(V(G), E(G))$ be a simple graph and let $\delta$ be the minimum degree of $G$. If $S p(G)=S p\left(F_{p, q}\right)$ with positive integers $p \geq 2$ and $q \geq 2$, then the following three properties hold.

(i). $|V(G)|=p q+1$;

(ii). $G$ is connected;

(iii). $2 \leq \delta \leq q+1$.

Proof. (i) is obvious and (ii) follows from the fact that the number of components of $G$ is equal to the multiplicity of 0 .

(iii). Let $x \in V(G)$ with $d_{x}=\delta$. By (11) in Lemma 2.3, we have

$$
\frac{q-1}{q^{2}} d_{x}<\widehat{d}_{x}=\sum_{w \sim x} \frac{1}{d_{w}} \leq 1 .
$$

Hence $d_{x}<\frac{q^{2}}{q-1} \leq q+2$. So $\delta=d_{x} \leq q+1$ since $\delta$ is integer. By (ii), $d(x)=\delta \geq 1$. So there exists a vertex $u \in V(G)$ such that $u \sim x$. By (21) in Lemma 2.3, $\widehat{\lambda}_{u x}>0$. Then there exists another vertex $w \neq u, x$ such that it is adjacent to both $u$ and $x$. So $\delta \geq 2$.

Lemma 2.5. Let $G=(V(G), E(G))$ be a simple graph with $m$ edges and the minimum degree $\delta$ such that $S p(G)=S p\left(F_{p, q}\right)$ with positive integers $p \geq 2$ and $q \geq 2$. If $d_{x}=\delta$ for $x \in V(G)$ with the neighbor set $N(x)=\left\{y_{1}, \ldots, y_{\delta}\right\}$ and $d_{y_{1}} \leq \cdots \leq d_{y_{\delta}}$, then $d_{y_{1}}=\delta$.

Proof. We consider the following two cases.

Case 1: $d_{y_{1}} \geq \delta+2$. By (11) in Lemma 2.3, we have

$$
\frac{(q-1) \delta}{q^{2}}=\frac{(q-1) d_{x}}{q^{2}}<\widehat{d}_{x}=\sum_{w \sim x} \frac{1}{d_{w}} \leq \frac{\delta}{\delta+2},
$$

which implies $\delta<q-1+\frac{1}{q-1}$. So $\delta \leq q-1$ by $q \geq 2$. On the other hand, by (2) in Lemma 2.3, we have

$$
\frac{q-2}{q}<\widehat{\lambda}_{x y_{1}}=\sum_{w \sim x, w \sim y_{1}} \frac{1}{d_{w}} \leq \frac{\delta-1}{\delta+2},
$$

which implies $\delta>\frac{3 q-4}{2}$. Hence $q-1 \geq \delta>\frac{3 q-4}{2}$, i.e., $q<2$. A contradiction.

Case 2: $d_{y_{1}}=\delta+1$. Then $\left|N(x) \bigcap N\left(y_{1}\right)\right|=\delta-1$. In fact, suppose that $\left|N(x) \cap N\left(y_{1}\right)\right|<\delta-1$. by (2) in Lemma 2.3, we have

$$
\frac{q-2}{q}<\widehat{\lambda}_{x y_{1}}=\sum_{w \sim x, w \sim y_{1}} \frac{1}{d_{w}} \leq \frac{\delta-2}{\delta+1},
$$


which implies that $\frac{3 q-2}{2}<\delta$. On the other hand, by (11) in Lemma 2.3, we have

$$
\frac{(q-1) d_{x}}{q^{2}}<\widehat{d}_{x}=\sum_{i=1}^{\delta} \frac{1}{d_{y_{i}}} \leq \frac{\delta}{\delta+1},
$$

which implies $\delta<q+\frac{1}{q-1}$. So $\delta \leq q$ by $q \geq 2$. Hence $\frac{3 q-2}{2}<\delta \leq q$, i.e., $q<2$. This is a contradiction. So $\left|N(x) \bigcap N\left(y_{1}\right)\right|=\delta-1$, i.e, $N(x) \cap N\left(y_{1}\right)=$ $\left\{y_{2}, \ldots, y_{\delta}\right\}$. Then by (11) and (2) in Lemma 2.3, we have

$$
\begin{aligned}
& \frac{(q+1) d_{x}^{2}}{2 m q^{2}}+\frac{(q-1) d_{x}}{q^{2}}=\sum_{i=1}^{\delta} \frac{1}{d_{y_{i}}} \\
& \frac{(q+1) d_{x} d_{y_{1}}}{2 m q^{2}}+\frac{(q-2)}{q}=\sum_{i=2}^{\delta} \frac{1}{d_{y_{i}}} .
\end{aligned}
$$

Hence by (41) and $\delta+1=d_{y_{1}} \leq \cdots \leq d_{y_{\delta}}$, we have

$$
\frac{(q-1) \delta}{q^{2}}<\sum_{i=1}^{\delta} \frac{1}{d_{y_{i}}} \leq \frac{\delta}{\delta+1} .
$$

Thus $\delta<q+\frac{1}{q-1}$, i.e., $\delta \leq q$ by $q \geq 2$. On the other hand, by (15), we have $\frac{q-2}{q}<\frac{\delta-1}{\delta+1}$, which implies that $q<\delta+1$, i.e., $q \leq \delta$. Hence $\delta=q \geq 2$. Furthermore, by $d_{x}=\delta=q$, subtracting (5) from (44) yields $2 m=(\delta+1)^{2}$. Since $d_{y_{2}} \geq d_{y_{1}} \geq \delta+1$, there exists a vertex $w \notin\left\{x, y_{1}, \ldots, y_{\delta}\right\}$ with $d_{w} \geq$ $\delta \geq 2$ Hence $(\delta+1)^{2}=2 m \leq \delta+(\delta+1) \delta+2=(\delta+1)^{2}+1$, which is a contradiction.

Therefore by Cases 1 and 2, we have $d_{y_{1}}=\delta$ and the assertion holds.

Lemma 2.6. Let $G=(V(G), E(G))$ be a simple graph with $m$ edges and the minimum degree $\delta$ such that $S p(G)=S p\left(F_{p, q}\right)$ for positive integers $p \geq 2$ and $q \geq 2$. If $d_{x}=\delta$ for $x \in V(G)$ with the neighbor set $N(x)=\left\{y_{1}, \ldots, y_{\delta}\right\}$ and $d_{y_{1}} \leq \cdots \leq d_{y_{\delta}}$, then $N(x) \bigcap N\left(y_{1}\right)=\left\{y_{2}, \ldots, y_{\delta}\right\}$.

Proof. By Lemma 2.5, $d_{y_{1}}=\delta$. Now we consider the following two cases.

Case 1: $\left|N(x) \bigcap N\left(y_{1}\right)\right| \leq \delta-3$. By (2) in Lemma 2.3, we have

$$
\frac{q-2}{q}<\widehat{\lambda}_{x y_{1}}=\sum_{w \sim x, w \sim y_{1}} \frac{1}{d_{w}} \leq \frac{\delta-3}{\delta}
$$

which yields that $\frac{3 q}{2}<\delta$. By Lemma 2.4(iii), we get $\frac{3 q}{2}<\delta \leq q+1$, i.e., $q<2$. This contradicts to the condition.

Case 2: $\left|N(x) \bigcap N\left(y_{1}\right)\right|=\delta-2$. By (2) in Lemma 2.3, we have $\frac{q-2}{q}<\frac{\delta-2}{\delta}$, which yields $q<\delta$, i.e., $q \leq \delta-1$. By Lemma 2.4(iii), we have $\delta=q+1$. By (1) and (2) in Lemma 2.3, we have

$$
\frac{(q+1) d_{x}^{2}}{2 m q^{2}}+\frac{(q-1) d_{x}}{q^{2}}=\sum_{i=1}^{\delta} \frac{1}{d_{y_{i}}},
$$




$$
\frac{(q+1) d_{x} d_{y_{1}}}{2 m q^{2}}+\frac{q-2}{q}=\sum_{i=2}^{\delta} \frac{1}{d_{y_{i}}}-\frac{1}{d_{y_{j}}} \text { for some } 2 \leq j \leq \delta .
$$

By $d_{x}=\delta=q+1$ and $d_{y_{1}}=\delta$, subtracting (7) from (66) yields

$$
\frac{(q-1)(q+1)}{q^{2}}-\frac{q-2}{q}=\frac{1}{q+1}+\frac{1}{d_{y_{j}}} .
$$

Hence $d_{y_{j}}=q+\frac{q}{q^{2}+q-1}$ contradicts the fact that $d_{y_{j}}$ is an integer.

Hence by Cases 1 and 2, we have $\left|N(x) \bigcap N\left(y_{1}\right)\right|=\delta-1$ and the assertion holds.

Lemma 2.7. Let $G=(V(G), E(G))$ be a simple graph with $m$ edges and the minimum degree $\delta$ such that $S p(G)=S p\left(F_{p, q}\right)$ for positive integers $p \geq 2$ and $q \geq 2$. If $d_{x}=\delta$ for $x \in V(G)$ with the neighbor set $N(x)=\left\{y_{1}, \ldots, y_{\delta}\right\}$ and $\delta=d_{x}=d_{y_{1}}=\cdots=d_{y_{k}}<d_{y_{k+1}} \leq \cdots \leq d_{y_{\delta}}$ for $1 \leq k \leq \delta-1$, then $N(x) \cap N\left(y_{i}\right)=\left\{y_{1}, \ldots, y_{i-1}, y_{i+1}, \ldots, y_{\delta}\right\}$ for $i=1, \ldots, k$ and $d_{y_{k+1}} \geq \delta+2$.

Proof. By Lemma 2.6 and $\delta=d_{x}=d_{y_{1}}=\cdots=d_{y_{k}}$, it is easy to see that $N(x) \bigcap N\left(y_{i}\right)=\left\{y_{1}, \ldots, y_{i-1}, y_{i+1}, \ldots, y_{\delta}\right\}$ for $i=1, \ldots, k$. Now we prove that $d_{y_{k+1}} \geq \delta+2$. Suppose that $d_{y_{k+1}}<\delta+2$. Then $d_{y_{k+1}}=\delta+1$ by $d_{y_{k+1}}>d_{y_{k}}=\delta$. We consider the following three cases.

Case 1: $\left|N(x) \cap N\left(y_{y_{k+1}}\right)\right| \leq \delta-3$. By (2) in Lemma 2.3, we have

$$
\frac{q-2}{q}<\widehat{\lambda}_{x y_{k+1}}=\sum_{w \sim x, w \sim y_{k+1}} \frac{1}{d_{w}} \leq \frac{\delta-3}{\delta},
$$

which implies that $\delta>\frac{3 q}{2}$. By Lemma 2.4 (iii), $\delta \leq q+1$. Thus we have $q+1>\frac{3 q}{2}$, i.e., $q<2$. A contradiction.

Case 2: $\left|N(x) \bigcap N\left(y_{y_{k+1}}\right)\right|=\delta-2$. By (2) in Lemma 2.3, we have

$$
\frac{q-2}{q}<\widehat{\lambda}_{x y_{k+1}} \leq \frac{\delta-2}{\delta}
$$

Then $q<\delta$, i.e., $q+1 \leq \delta$. By Lemma 2.4 (iii), $\delta \leq q+1$. Thus we have $\delta=q+1$. By (11) and (2) in Lemma 2.3, we have

$$
\frac{(q+1) d_{x}^{2}}{2 m q^{2}}+\frac{(q-1) d_{x}}{q^{2}}=\sum_{i=1}^{\delta} \frac{1}{d_{y_{i}}}
$$

and

$$
\frac{(q+1) d_{x} d_{y_{k+1}}}{2 m q^{2}}+\frac{q-2}{q}=\sum_{i=1}^{\delta} \frac{1}{d_{y_{i}}}-\frac{1}{d_{y_{k+1}}}-\frac{1}{d_{j}} \text { for some } k+2 \leq j \leq \delta .
$$

By $d_{x}=\delta=q+1$ and $d_{y_{k+1}}=\delta+1=q+2$, subtracting (9) from (8) yields that

$$
\frac{1}{d_{y_{j}}}=\frac{q^{2}+3 q-2}{q^{2}(q+2)}-\frac{(q+1)^{2}}{2 m q^{2}}
$$


Since $2 m \geq d_{x}+d_{y_{1}}+\cdots+d_{y_{\delta}}>\delta(\delta+1)=(q+1)(q+2)$, we have

$$
\frac{1}{d_{y_{j}}}>\frac{q^{2}+3 q-2}{q^{2}(q+2)}-\frac{(q+1)^{2}}{(q+1)(q+2) q^{2}} .
$$

So $d_{y_{j}}<q+2=\delta+1$ contradicts that $d_{y_{j}} \geq d_{y_{k+1}}=\delta+1$.

Case 3: $\left|N(x) \bigcap N\left(y_{y_{k+1}}\right)\right|=\delta-1$. Then $N(x) \bigcap N\left(y_{y_{k+1}}\right)=\left\{y_{1}, \ldots, y_{k}\right.$, $\left.y_{k+2}, \ldots, y_{\delta}\right\}$. By (11) and (2) in Lemma 2.3, we have

$$
\begin{gathered}
\frac{(q+1) d_{x}^{2}}{2 m q^{2}}+\frac{(q-1) d_{x}}{q^{2}}=\sum_{i=1}^{\delta} \frac{1}{d_{y_{i}}} \\
\frac{(q+1) d_{x} d_{y_{k+1}}}{2 m q^{2}}+\frac{q-2}{q}=\sum_{i=1}^{\delta} \frac{1}{d_{y_{i}}}-\frac{1}{d_{y_{k+1}}} .
\end{gathered}
$$

Subtracting (11) from (10) yields that

$$
\frac{(q-1) \delta}{q^{2}}=\frac{q-2}{q}+\frac{1}{\delta+1}+\frac{(q+1) \delta}{2 m q^{2}} .
$$

By $\delta \leq q+1$ in Lemma 2.4 and (12), we have

$$
\frac{(q-1) \delta}{q^{2}}>\frac{q-2}{q}+\frac{1}{q+2},
$$

which implies $\delta>q-1$ by $q \geq 2$. Hence $\delta=q$ or $\delta=q+1$. We consider the following two subcases.

Subcase 3.1: $\delta=q$. By (12), we have

$$
2 m=(q+1)^{2}
$$

Furthermore, by (10), we have

$$
\frac{1}{q+1}+\frac{q-1}{q}=\sum_{i=1}^{\delta} \frac{1}{d_{y_{i}}} \leq \frac{k}{\delta}+\frac{\delta-k}{\delta+1} .
$$

Thus $k \geq \delta-1$ and $k=\delta-1$. Since $d_{k+1}=\delta+1$, there exists a vertex $z \in$ $V(G) \backslash\left\{x, y_{1}, \ldots, y_{\delta}\right\}$ such that $z \sim y_{\delta}$. Furthermore, $N(z) \bigcap\left\{x, y_{1}, \ldots, y_{\delta-1}\right\}=$ $\emptyset$. So there exists another vertex $w \in V(G) \backslash\left\{x, y_{1}, \ldots, y_{\delta}, z\right\}$ by $d_{z} \geq \delta \geq 2$. Therefore, $2 m \geq \delta^{2}+(\delta+1)+d_{z}+d_{w}>(\delta+1)^{2}=(q+1)^{2}$ which contradicts to $2 m=(q+1)^{2}$.

Subcase 3.2: $\delta=q+1$. By (12), we have

$$
2 m=\frac{(q+1)^{2}(q+2)}{q^{2}+3 q-2}<2 q+2 .
$$

It is a contradiction. Hence $d_{y_{k+1}} \geq \delta+2$ and the assertion holds. 
Lemma 2.8. Let $G=(V(G), E(G))$ be a simple graph with $m$ edges and the minimum degree $\delta$ such that $S p(G)=S p\left(F_{p, q}\right)$ for positive integers $p \geq 2$ and $q \geq 2$. If $d_{x}=\delta$ for $x \in V(G)$ with the neighbor set $N(x)=\left\{y_{1}, \ldots, y_{\delta}\right\}$ and $\delta=d_{x}=d_{y_{1}}=\cdots=d_{y_{k}}<d_{y_{k+1}} \leq \cdots \leq d_{y_{\delta}}$ for some $1 \leq k \leq \delta-1$, then

(i) $\delta=q$.

(ii) $N(x) \bigcap N\left(y_{k+1}\right)=\left\{y_{1}, \cdots, y_{k}, y_{k+2}, \cdots, y_{\delta}\right\}$.

(iii) $k=q-1$.

(iv) $d_{y_{q}}=p q$ and $d_{w}=q$ for $w \in V(G) \backslash\left\{y_{q}\right\}$.

(v). $2 m=p q(q+1)$.

Proof. (i). By Lemma 2.7, we have $N(x) \bigcap N\left(y_{k}\right)=\left\{y_{1}, \cdots, y_{k-1}, y_{k+1}, \cdots, y_{\delta}\right\}$. Hence by (1) and (2) in Lemma 2.3, we have

$$
\begin{gathered}
\frac{(q+1) d_{x}^{2}}{2 m q^{2}}+\frac{(q-1) d_{x}}{q^{2}}=\sum_{i=1}^{\delta} \frac{1}{d_{y_{i}}}, \\
\frac{(q+1) d_{x} d_{y_{k}}}{2 m q^{2}}+\frac{q-2}{q}=\sum_{i=1}^{\delta} \frac{1}{d_{y_{i}}}-\frac{1}{d_{y_{k}}} .
\end{gathered}
$$

By $d_{x}=d_{y_{k}}=\delta$, subtracting (14) from (13) yields $\frac{(q-1) \delta}{q^{2}}-\frac{q-2}{q}=\frac{1}{\delta}$. So $\delta=q$ and (i) holds.

(ii). Suppose that $\left|N(x) \bigcap N\left(y_{k+1}\right)\right| \leq \delta-2$. Then by (2) in Lemma 2.3, we have

$$
\frac{q-2}{q}<\widehat{\lambda}_{x y_{k+1}}=\sum_{w \sim x, w \sim y_{k+1}} \frac{1}{d_{w}} \leq \frac{\delta-2}{\delta} .
$$

So $q<\delta$, which contradicts to (i). So $\left|N(x) \bigcap N\left(y_{k+1}\right)\right|=\delta-1$ and (ii) holds.

(iii). By (2) in Lemma 2.3 and (ii), we have

$$
\begin{gathered}
\frac{(q+1) d_{x} d_{y_{k}}}{2 m q^{2}}+\frac{q-2}{q}=\sum_{i=1}^{\delta} \frac{1}{d_{y_{i}}}-\frac{1}{d_{y_{k}}} \\
\frac{(q+1) d_{x} d_{y_{k+1}}}{2 m q^{2}}+\frac{q-2}{q}=\sum_{i=1}^{\delta} \frac{1}{d_{y_{i}}}-\frac{1}{d_{y_{k+1}}} .
\end{gathered}
$$

Subtracting (15) from (16) yields

$$
\frac{(q+1) d_{x}\left(d_{y_{k+1}}-d_{y_{k}}\right)}{2 m q^{2}}=-\frac{1}{d_{y_{k+1}}}+\frac{1}{d_{y_{k}}} .
$$

Moreover, by $d_{y_{k+1}}>d_{y_{k}}=d_{x}=\delta=q$ from (ii), we have $2 m=(q+1) d_{y_{k+1}}$. Furthermore, by (1) in Lemma 2.3,

$$
\frac{(q+1) d_{x}^{2}}{2 m q^{2}}+\frac{(q-1) d_{x}}{q^{2}}=\sum_{i=1}^{\delta} \frac{1}{d_{y_{i}}} \leq \frac{k}{\delta}+\frac{\delta-k}{d_{y_{k+1}}}
$$


By $2 m=(q+1) d_{y_{k+1}}$ and $d_{x}=\delta=q$, we have

$$
\frac{1}{d_{y_{k+1}}}+\frac{q-1}{q} \leq \frac{k}{q}+\frac{q-k}{d_{y_{k+1}}}
$$

i.e., $(q-k-1)\left(d_{y_{k+1}}-q\right) \leq 0$. So $k \geq q-1=\delta-1$. Hence $k=\delta-1=q-1$ and (iii) holds.

(iv). By (iii), $2 m=(q+1) d_{y_{\delta}}$. Moreover, by Lemma 2.4(i), $|V(G)|=p q+1$. Hence

$$
(\delta+1) d_{y_{\delta}}=2 m=d_{y_{\delta}}+\sum_{w \neq y_{\delta}} d_{w} \geq d_{y_{\delta}}+\delta(p q) .
$$

Hence $d_{y_{\delta}} \geq p q$. However, $d_{y_{\delta}} \leq|V(G)|-1=p q$. So $d_{y_{\delta}}=p q$. Furthermore, (17) becomes equality, which implies $d_{w}=\delta$ for $w \neq y_{\delta}$. So (iv) holds.

(v). By (i), (iii) and (iv), we have $q=\delta, 2 m=(q+1) d_{y_{\delta}}$ and $d_{y_{\delta}}=p q$, which implies $2 m=(q+1) p q$.

Now we are ready to prove the main theorem in this paper.

Proof of Theorem 1.1. If $p=1$, then $F_{1, q}=K_{q+1}$, so it is determined by its normalized Laplacian spectrum. If $q=1$ and $p \leq 2$, or $q=1$ and $p \geq 3$, the assertion follows from Lemma 2.2 .

Now we assume that $p \geq 2$ and $q \geq 2$. Let $G$ be any graph with $\operatorname{Sp}(G)=$ $S p\left(F_{p, q}\right)$. Then $G$ is a connected graph on $p q+1$ vertices by Lemma 2.4. Furthermore let $d_{x}=\delta(G)$ for $x \in V(G)$ and $N(x)=\left\{y_{1}, \ldots, y_{\delta}\right\}$ with $d_{y_{1}} \leq$ $\cdots \leq d_{y_{\delta}}$. By Lemma 2.5, $d_{y_{1}}=\delta$. Hence we assume that $\delta=d_{y_{1}}=\cdots=$ $d_{y_{k}}<d_{y_{k+1}} \leq \cdots \leq d_{y_{\delta}}, 1 \leq k \leq \delta$. Then $1 \leq k \leq \delta-1$, otherwise $G=K_{\delta+1}$ has only two distinct $\mathcal{L}$ - eigenvalues. Hence by Lemma 2.8, we have $\delta=q$, $d_{y_{1}}=\cdots=d_{y_{q-1}}=q$ and the induced subgraph $G\left[x, y_{1}, \cdots, y_{q-1}\right]$ by vertex set $\left\{x, y_{1}, \cdots, y_{q-1}\right\}$ is a clique of order $q$. Furthermore, $y_{q}$ is adjacent to each vertex in $G$. For any $u \in V(G) \backslash\left\{x, y_{1}, \ldots, y_{q}\right\}$, then $u$ is not adjacent to $x, y_{1}, \ldots, y_{q-1}$. By (3) in Lemma 2.3, we have

$$
\widehat{\mu}_{u x}=\sum_{w \sim u, w \sim v} \frac{1}{d_{w}}=\frac{1}{d_{y_{q}}}=\frac{(q+1) d_{u} d_{x}}{2 m q^{2}} .
$$

By Lemma 2.8, $d_{u}=q=\delta$, i.e., the degree of each vertex except $y_{q}$ in $V(G)$ is $q$. Choosing any vertex $v \in V(G) \backslash\left\{x, y_{1}, \ldots, y_{q}\right\}$, let $N(v)=\left\{z_{1}, \ldots, z_{q-1}, y_{q}\right\}$ since $d_{v}=q$ and $y_{q} \sim v$. Then $d_{z_{1}}=\cdots=d_{z_{q-1}}=q$ and $N(z) \bigcap\left\{x, y_{1}, \ldots, y_{q}\right\}=$ $\left\{y_{q}\right\}$. By (2) in Lemma 2.3, we have

$$
\frac{(q+1) d_{v} d_{z_{i}}}{2 m q^{2}}+\frac{q-2}{q}=\sum_{w \sim v, w \sim z_{i}} \frac{1}{d_{w}}=\frac{1}{d_{y_{q}}}+\frac{\left|N(v) \bigcap N\left(z_{i}\right) \backslash\left\{y_{q}\right\}\right|}{q}
$$

for $v \sim z_{i}$. Since $2 m=p q(q+1), d_{v}=d_{z_{i}}=q$ and $d_{y_{q}}=p q$, we have $\left|N(v) \bigcap N\left(z_{i}\right) \backslash\left\{y_{q}\right\}\right|=q-1$. Hence the induced subgraph $G\left[v, z_{1}, \ldots, z_{q-1}\right]$ by vertex set $\left\{v, z_{1}, \ldots, z_{q-1}\right\}$ is a clique of order $q$. By repeating the above process, $G$ must be $F_{p, q}$. So the assertion holds.

Acknowledgement. The authors would like to thank the referee for very constructive suggestions and comments on this paper. 


\section{References}

[1] S. Butler, Algebraic aspects of the normalized Laplacian. Recent trends in combinatorics, 295-315, IMA Vol. Math. Appl., 159, Springer, 2016

[2] S. Butler and J. Grout, A construction of cospectral graphs for the normalized Laplacian. Electron. J. Combin. 18 (2011), no. 1, Paper 231, 20 pp.

[3] S. Butler and K. Heysse, A cospectral family of graphs for the normalized Laplacian found by toggling. Linear Algebra Appl. 507(2016) 499-512.

[4] F. Chung, Spectral Graph Theory, 2nd edn. AMS, Providence, 1997.

[5] S. M. Cioabă, W. H. Haemers, J. Vermette and W. Wong, The graphs with all but two eigenvalues equal to \pm 1 , J. Algebr. Comb. 41(2015) 887897

[6] P. Erdős, A. Rényi and V. Sós, On a problem of graph theory. Studia Sci. Math. Hung. 1 (1966) 215-235.

[7] Y.-Q. Lin, J.-L. Shu and Y. Meng, Laplacian spectrum characterization of extensions of vertices of wheel graphs and multi-fan graphs. Comput. Math. Appl. 60 (2010) 2003-2008.

[8] X.-G. Liu,Y.-P. Zhang and X.-Q. Gui, The multi-fan graphs are determined by their Laplacian spectra. Discrete Math. 308 (2008) 4267-4271.

[9] E. R. Van Dam and W. H. Haemers, Which graphs are determined by their spectrum?, Linear Algebra Appl., 373 (2003) 241-272.

[10] E. R. Van Dam and W. H. Haemers, Developments on spectral characterizations of graphs, Discrete Math., 309 (2009) 576-586.

[11] E. R. Van Dam and G. R. Omidi, Graphs whose normalized Laplacian has three eigenvalues. Linear Algebra Appl. 435 (2011) 2560-2569.

[12] J.-F. Wang, F. Belardo, Q.-X. Huang and B. Borovicanin, On the two largest Q-eigenvalues of graphs. Discrete. Math. 310 (2010) 2858-2866. 